\title{
Second-layer nucleation in coherent Stranski-Krastanov growth of quantum dots
}

\author{
José Emilio Prieto* \\ Centro de Microanálisis de Materiales, Departamento de Física de la Materia Condensada and Instituto Universitario "Nicolás Cabrera", \\ Universidad Autónoma de Madrid, E-28049 Madrid, Spain \\ Ivan Markov ${ }^{\dagger}$ \\ Institute of Physical Chemistry, Bulgarian Academy of Sciences, 1113 Sofia, Bulgaria
}

(Received 27 July 2011; revised manuscript received 10 October 2011; published 4 November 2011)

\begin{abstract}
We have studied the monolayer-bilayer transformation in the case of the coherent Stranski-Krastanov growth. We have found that the energy of formation of a second-layer nucleus is largest at the center of the first-layer island and smallest on its corners. Thus nucleation is expected to take place at the corners (or the edges) rather than at the center of the islands as in the case of homoepitaxy. The critical nuclei have one atom in addition to a compact shape, which is either a square of $i \times i$ or a rectangle of $i \times(i-1)$ atoms, with $i>1$ an integer. When the edge of the initial monolayer island is much larger than the critical nucleus size, the latter is always a rectangle plus an additional atom, adsorbed at the longer edge, which gives rise to a new atomic row in order to transform the rectangle into the equilibrium square shape.
\end{abstract}

DOI: 10.1103/PhysRevB.84.195417

PACS number(s): 68.35.Md, 68.43.Hn, 68.55.A-, 81.07.Ta

The Stranski-Krastanov (SK) mode of epitaxial growth is a nice example of an instability of planar, two-dimensional (2D) growth against three-dimensional (3D) islanding due to a nonzero lattice misfit between the deposit and the substrate materials. This leads to the formation of arrays of self-assembled small crystallites. In the case of semiconductor overgrowth, these are known as quantum dots and have important applications in optoelectronic devices. The physical reason for the occurrence of this $2 \mathrm{D}-3 \mathrm{D}$ transition is well established as the gain of strain energy at the expense of surface energy. ${ }^{1-8}$ However, the mechanism of formation of $3 \mathrm{D}$ islands on the planar wetting layer in the case of coherent (dislocationless) SK growth is still an unsolved problem in spite of intensive studies in the last two decades.

Voigtländer and Zinner ${ }^{9}$ observed by scanning tunneling microscopy (STM) that faceted 3D Ge islands form at the same locations on a $\mathrm{Si}(111)$ surface at which $2 \mathrm{D}$ islands were observed in the initial stage of deposition immediately after exceeding the critical thickness of the wetting layer. Ebiko et al. ${ }^{10}$ found that the scaling function of the volume distribution of 3D InAs quantum dots on the surface of GaAs coincide with the scaling function for 2D submonolayer homoepitaxy with critical cluster size $i=1$. Mo et al. ${ }^{11}$ observed $\mathrm{Ge}$ islands representing elongated pyramids (hut clusters) bounded by $\{105\}$ facets inclined by $11.3^{\circ}$ to the substrate. The authors suggested that the hut clusters are a step in the pathway to the formation of larger islands with steeper side walls known in the literature as (rounded) domes and (faceted) barns. ${ }^{12}$ Chen et al. ${ }^{13}$ studied the earliest stages of Ge islanding on $\mathrm{Si}(001)$ and established that $\mathrm{Ge}$ islands smaller than the hut clusters are not bounded by discrete $\{105\}$ facets. This result was later confirmed by Vailionis et al. ${ }^{14}$ who observed the formation of three to four monolayers (ML)-high prepyramids with rounded bases, which exist over a narrow interval of a Ge coverage in the beginning of the 2D-3D transition. Also Arapkina and Yuryev found that the formation of the second layer of Ge clusters results in rearrangement of the first layer. ${ }^{15}$ Sutter and Lagally ${ }^{16}$ observed by low-energy electron microscopy (LEEM) the formation of an array of stepped mounds (ripples) as precursors of the hut clusters on the surface of low misfit alloyed SiGe films on $\mathrm{Si}(001)$, which are inherent to strained films. ${ }^{17-19}$

An insight concerning the formation of 3D islands on top of the wetting layer came from Tersoff and LeGoues who suggested a nucleation mechanism as the result of the interplay between the positive surface energy of the islands and the relaxation of the strain energy in the islands relative to that of the wetting layer. ${ }^{2}$ A critical volume is thus defined beyond which irreversible 3D growth takes place. It was found that the energetic barrier associated with the critical volume is proportional to $f^{-4}$ where $f$ is the lattice misfit. This concept, although very attractive, does not give any information about the mechanism of formation of the 3D islands. On the contrary, based on their observations Sutter and Lagally suggested that $3 \mathrm{D}$ islands could be formed without the necessity of overcoming an energetic barrier. ${ }^{16}$ Similar views and further elaboration of the idea of barrierless transformation of the ripples into faceted islands were suggested by Tromp et al. ${ }^{20}$ and by Tersoff et $a .^{21}$

Priester and Lannoo, on the basis of microscopic calculations within the Keating model, suggested that 2D islands appear as precursors of the $3 \mathrm{D}$ islands. ${ }^{22}$ Korutcheva et al ${ }^{23}$ and Prieto and Markov ${ }^{24}$ established on the base of $1+1$ dimensional models that the minimum energy pathway of the 2D-3D transition has to consist of a sequence of states with thickness increasing by a single ML and that are stable in separate intervals of volume. The first step in this process is the rearrangement of monolayer into bilayer islands. This result was later confirmed by a $2+1$ dimensional model. ${ }^{25}$ Khor and Das Sarma ${ }^{26}$ and Xiang et al. ${ }^{27}$ reached the same conclusion by Monte Carlo simulations. The first authors found that during growth, the material for the bilayer island originates almost completely from the initial ML-high island in addition to some small amount coming from the vapor phase, the material for the three-ML island comes from the original bilayer island, etc. This layer-by-layer mechanism of growth takes place if 
the deposited material is sufficiently stiff. ${ }^{28}$ If the deposited material is soft enough, such as $\mathrm{Pb}^{29}$ or $\mathrm{In},{ }^{30}$ the ML islands are expected to transform directly into multilayer islands with preferred heights (process known in the literature as electronic growth $^{31}$ ) because of the requirement of greater thickness to give rise to the necessary amount of strain relaxation, as was theoretically predicted in Ref. 28.

All these results are in accordance with the finding of Stoyanov and Markov $^{32}$ who established for the case of Volmer-Weber growth at zero misfit that an increase of the cluster volume gives rise to the stability of increasingly higher islands. The islands increase in height by one ML beyond certain critical volumes. The process of transformation of $n$-ML thick to $(n+1)$-ML thick islands is described by a curve giving the energy as a function of the number of atoms transferred to the upper level, which is characteristic for a nucleation process. It displays a maximum at a certain number of atoms and then decreases steadily up to the end of the transformation. The atoms of the $(n+1)$ th $\mathrm{ML}$ are detached from the edges of the lower monolayer island. The chemical potentials of the critical nucleus and the cluster underneath are equal, which is an indication of a true nucleation process. More details can be found in Ref. 33.

Recently, Villain noted that in highly mismatched epitaxy, second-layer nuclei are expected to form close to the edges of the initial ML islands rather than at their centers. ${ }^{34}$ The reason is that the misfit strain is relaxed at the steps and "...atoms are happy to be there, because they find an atomic distance which is closer to the atomic distance they would like to have." 34 Thus, for sufficiently large values of the misfit, upper-layer nuclei will form at the edges, while at small misfits, the adatom concentration will be highest at the island's center and upperlayer nuclei will form preferentially there, as in the case of homoepitaxial growth. ${ }^{35}$ The presence of Ehrlich-Schwoebel barriers for interlayer diffusion can also be important. This however leads to the formation of growth pyramids rather than to quasi-equilibrium $3 \mathrm{D}$ crystallites. ${ }^{36}$

In the present paper we study the formation of second-layer nuclei at different locations on the first-layer island: center, edge, and corner (see Fig. 1). The model has been described in detail elsewhere. ${ }^{25}$ Briefly, we consider an atomistic model in $2+1$ dimensions, in which the 3D crystallites have fcc structure and (100) surface orientation, thus possessing the shape of truncated square pyramids. The lattice misfit is the same in both orthogonal in-plane directions. We consider interactions only in the first coordination sphere. Inclusion of further coordination spheres is not expected to alter qualitatively the numerical results as long as epitaxial structures remain coherent. We perform a simple minimization procedure. The atoms interact through a pair potential containing two adjustable parameters $\mu$ and $v(\mu>v),{ }^{38,39}$

$$
V(r)=V_{0}\left[\frac{v}{\mu-v} e^{-\mu(r-b)}-\frac{\mu}{\mu-v} e^{-v(r-b)}\right],
$$

where $b$ is the equilibrium atom separation, For $\mu=2 v$ the above potential adopts the familiar Morse form. In spite of its simplicity, the above potential includes all necessary features to describe real materials (bonding strength and anharmonicity). The interatomic spacing of the substrate is
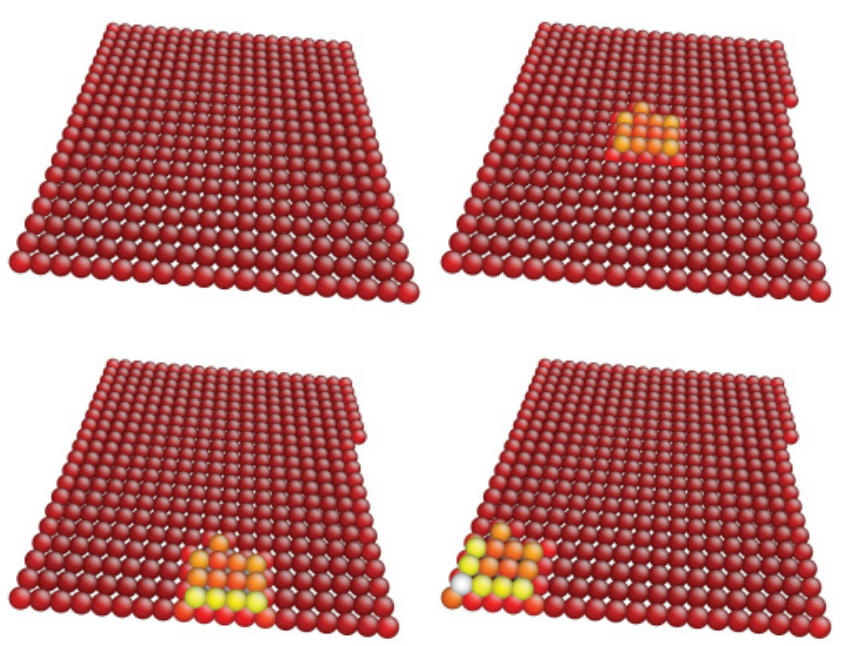

FIG. 1. (Color online) Locations of second-layer nuclei. From top to bottom and from left to right: initial $20 \times 20$ monolayer island; 13-atoms second-layer cluster nucleated at the terrace center, at an island edge, and at an island corner of the initial monolayer island. The color scale denotes the height of the considered atom and has been represented using the ATOMEYE software. ${ }^{37}$ This height is measured above the level of the corresponding crystallographic plane, but a constant fraction of the interlayer distance has been added in order to better distinguish atoms from different levels. The height is biggest at edges and corners due to the atoms climbing up on their neighbours underneath due to strain relaxation. The lattice misfit is $-7 \%$.

$a$ so the lattice misfit is given by $f=(b-a) / a$. The substrate is assumed to be rigid.

We study the transformation of monolayer into bilayer islands, considered as the first step of the 2D-3D transformation, along the procedure developed in Ref. 32. We assume the following model processes: The initial state is a square, ML-high island, whose size is larger than the critical value and that is thus unstable against monolayer to bilayer island transformation. ${ }^{25}$ Atoms detach from the edges of the initial ML island, diffuse on top of it and eventually aggregate and give rise to second-layer nuclei. These grow at the expense of the atoms detached from the edges of the lower island up to the moment when the upper island completely covers the lower level. To simulate this process, we detach atoms one by one from the edges of the lower island and arrange them on top, forming $2 \mathrm{D}$ clusters as compact as possible at one of three different locations: At the center of the terrace (center), at the center of one edge (edge), and at one of the corners (corner) of the initial monolayer island (see Fig. 1). The second-layer clusters are always as compact as possible [i.e., either a square of $i \times i$ or a rectangle of $i \times(i-1)$ atoms (where $i>1$ is an integer number)] plus eventually a fraction of an atomic row placed at a free edge of the island (at the longer edge in the case of a rectangular island). This mechanism is expected to describe most closely the experimental results, ${ }^{9-15}$ as discussed above.

The change in energy associated with the process of transformation at a particular stage is given by the difference between the total energies of the incomplete bilayer island and the initial monolayer island. We then compare the nucleation barriers and the number of atoms in the nuclei formed on 
the lower island center, edge, and corner locations. We find that, in addition to Villain's kinetic reasons, thermodynamics also plays in favor of a preferred formation of second-layer nuclei at the island's edges and corners where the strain is smaller.

As shown in Ref. 25, the sign of the misfit plays a crucial role in the mechanism of transformation of monolayer to bilayer islands. When the second-layer clusters are formed at the center of the first-layer islands, the curves for positive misfits show a true nucleation mechanism. The curves display sharp maxima followed by a decrease of the energy up to the complete transformation. The value at the maximum gives the energy barrier for nucleation of the upper layer. In the case of negative misfit, we observe a totally different mechanism. The transformation energy does not display a well-defined maximum but increases steadily up to a (relatively high) value beyond which it steeply collapses, close to the end of the transformation. The collapse is due to the coalescence of the single steps into bilayer facets, which possess a much smaller energy.
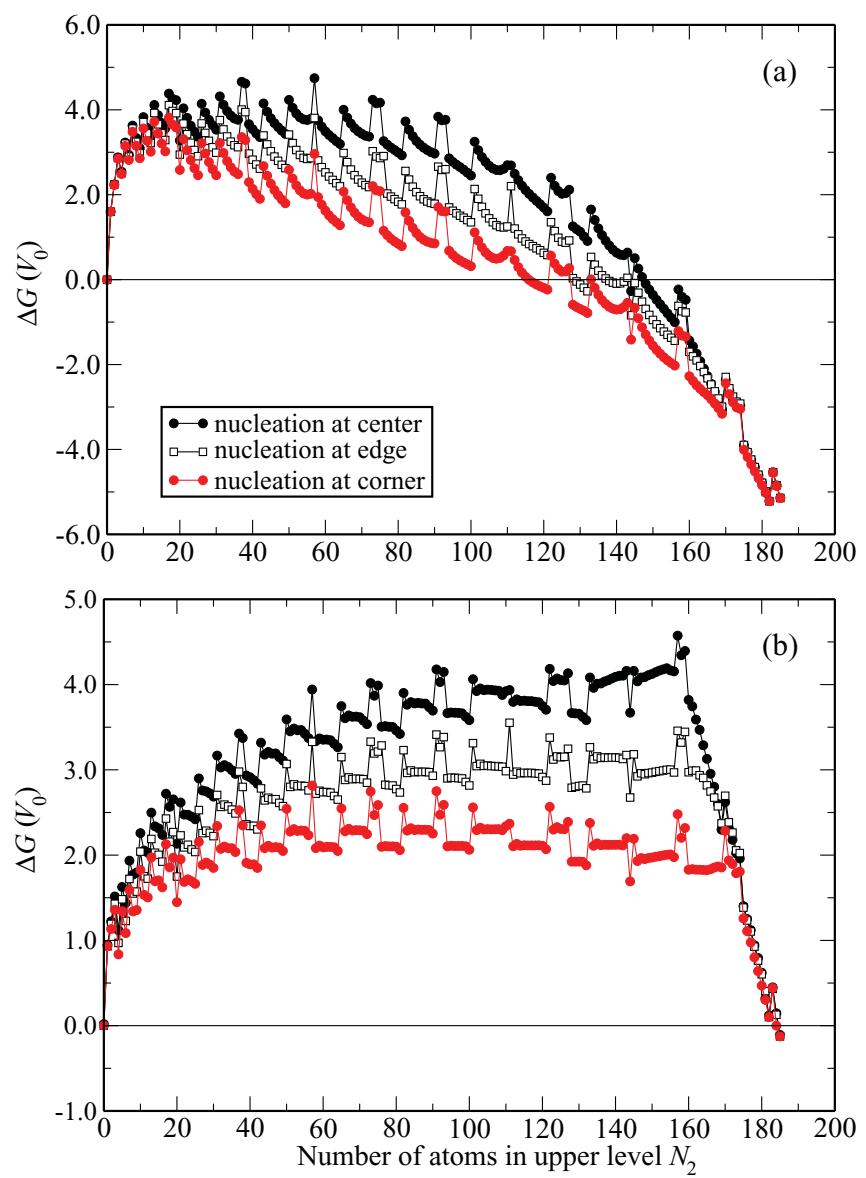

FIG. 2. (Color online) Transformation curves (total energy as a function of the number $N_{2}$ of atoms transferred to the second ML) for (a) positive $(+3.5 \%)$ and (b) and negative $(-12.0 \%)$ values of the misfit. A potential with $\mu=16$ and $v=14$ was used in the simulations and an initial monolayer island containing $20 \times 20=$ 400 atoms was considered. The second-layer islands are formed at the center, at one of the edges, and at one corner of the initial, monolayer island.
This behavior, different for different signs of the lattice misfit, is confirmed in Fig. 2 by the energy curves corresponding to nucleation at different locations on the initial square-shaped monolayer island containing 400 atoms. Figure 2 shows transformation curves for second-layer islands nucleated at the center of the monolayer island, at the middle of one of its edges and at one of its corners; the patterns of transformation assumed in the simulations is comparable in all cases, in particular second-layer clusters of similar shapes were chosen irrespective of their location on the initial monolayer island.

Figure 2 shows that, for both signs of the lattice misfit, the process of island nucleation at the corner position has the lowest energy barrier and the terrace-center position has the highest one, while the edge position shows an intermediate value. This can be understood in terms of the different level of strain relaxation at different positions of coherent islands. Atoms at the center of a terrace are forced by their lateral neighbours to adopt a similar lateral distance as atoms in the lower level and hence possess the highest strain. ${ }^{23,25}$ In contrast, atoms at edges and even more at corner positions have the possibility to displace laterally due to their reduced lateral coordination, so they relax epitaxial strain more efficiently. In this way, the process of $3 \mathrm{D}$ clustering is favoured by a higher degree of strain energy reduction in the cases of corner and edge nucleation. For negative values of the misfit (expanded overgrowths), the formation of second-layer islands on the first-layer islands' corners shows a slightly different behavior compared with the growth of islands at the terrace center [Fig. 2(b)]. In addition to the expected collapse of the energy at the end of the transformation, the energy displays broad maxima at a relatively large number of atoms. Thus we observe a superposition of the nucleationlike behavior and the collapse of the energy due to the coalescence of the single steps. Increasing the absolute value of the misfit leads to a decrease of the number of atoms in the critical nucleus.

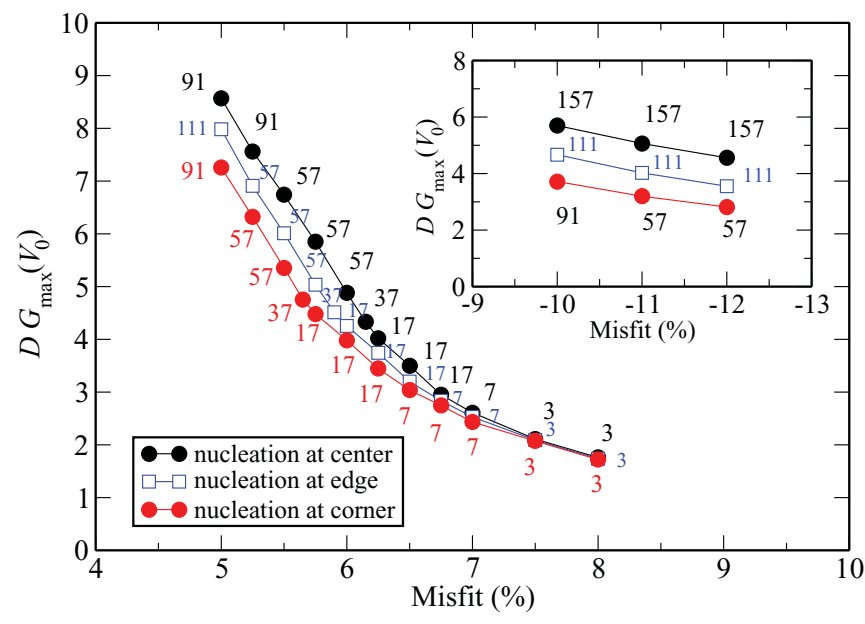

FIG. 3. (Color online) Heights of the nucleation barriers in units of $V_{0}$ as a function of the value of the lattice misfit (main plot: positive misfits; insert: negative misfits). The figures at each point denote the number of atoms, $n^{*}$, in the critical nucleus. The values for compressed islands were calculated for $\mu=2 v=12$, those for expanded islands for $\mu=16$ and $v=14$. A cluster size of 400 atoms was considered. 
Figure 3 shows our main result. It represents the heights of the nucleation barriers as a function of the lattice misfit both for positive and negative values and for the cases of second-layer nucleation at the center, edge, and corner of the initial monolayer island. The figures close to the data points give the number of atoms in the critical nuclei.

As seen in Fig. 3, for the case of compressed islands, small critical nucleus sizes and correspondingly small barrier heights are obtained for relatively small values of the lattice misfit, no larger than $8 \%$. Both magnitudes increase markedly with decreasing misfit value. In contrast, for expanded layers, much larger, unrealistically high absolute values of the lattice misfit, between 10 and $12 \%$, are required to obtain barriers of comparable heights. The effect is even stronger when considering that the calculations for negative misfits were performed for a stronger potential. Furthermore, the numbers of atoms in the critical nuclei is much larger than in compressed islands and the dependence of critical sizes and barrier heights on the absolute value of the misfit is also much less pronounced. The curves for nucleation at different locations show a clear energetic preference for corner as compared to center nucleation. As an example, for reasonably high positive values of the misfit (below 68\%), the difference in the barrier heights between center and corner second-level nucleation can be larger than the energy of a single atomic bond $\left(V_{0}\right.$ in our model), representing a decrease of roughly $25 \%$ or even more. This is of enormous significance given the exponential dependence of the nucleation rate on the barrier height. We conclude that for both signs of the lattice misfit and due to thermodynamic reasons, nucleation is expected to take place preferentially at the islands corners, followed by the edges, rather than at the islands centers.

Experimental evidence of the effect of misfit strain on second-layer nucleation has been obtained in organic [tetracene on $\mathrm{H}$-passivated $\mathrm{Si}(001)^{40}$ ] and in metal-on-metal systems such as $\mathrm{Pd} / \mathrm{Cu}(001) .{ }^{41}$ A further convincing example is the case of SK growth of $\operatorname{InAs}_{x} \mathrm{P}_{1-x}$ nanowires on InP. ${ }^{42}$ Images show that the second-layer clusters form preferentially on the upper side of steps, where maximum strain relaxation occurs. According to the authors, the explanation of this observation does not require accounting for the EhrlichSchwoebel effect. An explanation for this behavior can be of kinetic origin, based either on Villain's arguments or on the presence of Ehrlich-Schwoebel barriers (the adatom concentration has a maximum value at the step edges) $)^{43}$ or the thermodynamic reasons discussed in this paper. Most probably at least two of these factors act simultaneously.

Another interesting result follows from Fig. 3. The numbers $n^{*}$ of atoms in the critical nuclei always satisfy either the relation $n^{*}=i \times i+1$ or, more frequently, $n^{*}=i \times(i-$ $1)+1$, where $i$ is an integer giving the number of atoms in the longer edge of the rectangle. This means that the critical nuclei consist of a compact structure plus one additional atom. In the atomistic theory of nucleation on surfaces it is usually assumed that the critical nuclei contain one atom less than those necessary for a compact structure ${ }^{44}$ (for a review see Ref. 45). Thus the smallest nucleus (larger than 1 atom) on a surface with a square symmetry as in our case consists of three atoms located at the vertices of a rectangular triangle. The smallest stable cluster consists of four atoms located at the corners of a square. In order to disrupt a critical nucleus, one has to break a single bond whereas in order to disrupt the stable cluster, one has to simultaneously break two bonds. This explains the stability of the smallest stable cluster compared with the critical nucleus.

As noted by Kashchiev, ${ }^{46}$ the main difference between the classical nucleation theory (CNT) and the atomistic nucleation theory (ANT) is the nucleus shape. Whereas ANT allows any irregular shape that arises from the atomistic nature of the nucleus edges, in the CNT it is assumed that the nucleus possesses the equilibrium shape, which in our case [(100) surface and consideration of only first-neighbors interactions] is a square island. What we observe is a rectangle (with the shape closest to a square) plus one additional atom. The compact shape can be understood in terms of the highest coordination achieved. The question that arises is about the additional atom, which is the first in the new row.

Kaischew and Stranski derived expressions for the rate of $3 \mathrm{D}$ and $2 \mathrm{D}$ nucleation by using a completely kinetic approach. ${ }^{47,48}$ They argued that the barrier of formation of a $3 \mathrm{D}$ crystalline nucleus should include the energy of formation of a 2D nucleus on the side wall of a 3D cluster smaller by an atomic plane than the critical nucleus. Analogously, the barrier for 2D nucleation should include the barrier of formation of a new atomic row, which is in fact the barrier for attachment of the first atom of the row. The single atom gives birth to a new row of atoms thus playing the role of one-dimensional nucleus $^{49}$ (for a recent review see Ref. 50).

Considering the classical nucleation, Kaischew and Stranski also argued that the work of formation of a 3D nucleus with a cubic shape should include the work of formation of three 2D nuclei on neighboring crystal walls in order to transform the cube of $i$ atoms in its edge to a cube of $i+1$ atoms thus preserving the equilibrium shape. Analogously, the 2D square nucleus should build two rows of atoms on neighboring edges.

Kashchiev found that the nucleus size as a function of the supersaturation always satisfies the relation $n^{*}=i \times(i-$ 1) $+1,(i=2,3,4, \ldots)$, which means that the single additional atom begins the atomic row that transforms the rectangle $i \times$ $(i-1)$ into an island with the square equilibrium shape $i \times i{ }^{46}$ The calculation in which the work of formation of a $2 D$ nucleus is corrected by including the contribution of the additional atom is in much better agreement with the exact solution for the nucleation rate as derived by Becker and Döring in their seminal paper. ${ }^{51}$ Kashchiev found that only rectangular 2D critical nuclei form; the reason is that he considered them to grow on an infinitely large surface from an infinite vapor phase. On the contrary, we simulate the construction of 2D nuclei on a strained layer of finite size by removing the atoms from the edges of the lower island. In such a case, the detailed atomistics at the lower island also play a role.

This is demonstrated by the results shown in Fig. 4. Here, total energy curves were calculated both for a finite-sized initial island of $20 \times 20$ atoms and for a situation that simulates the same transformation sequence for an infinitely large initial monolayer island: The same configurations for the growing cluster in the second atomic level were considered, but in this second case, no atoms were detached from the first atomic level. The increasing total number of atoms was corrected by subtracting the calculated negative binding energy of an atom 


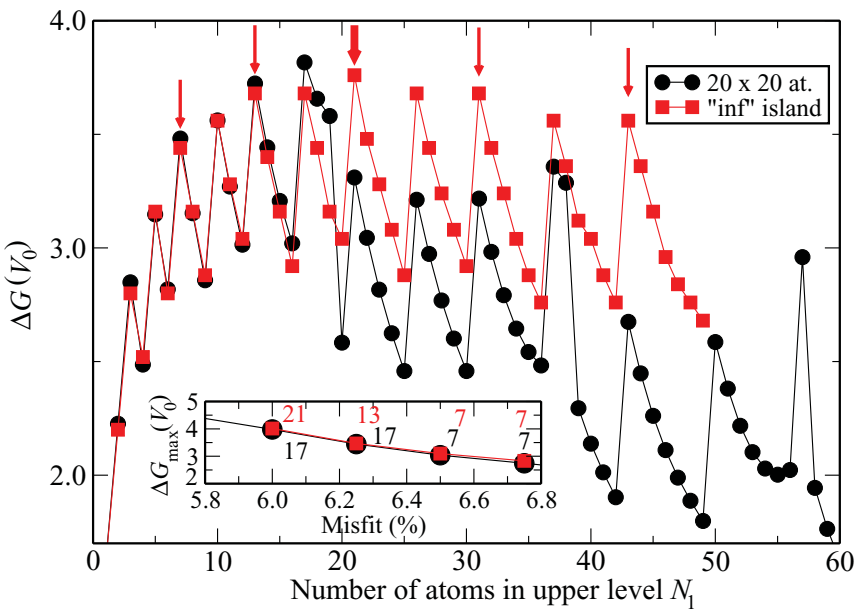

FIG. 4. (Color online) Closeup of the transformation curve for a lattice misfit of $+3.58 \%, \mu=16, v=14$, and a finite cluster of a size of 400 atoms (circles). Also shown (squares) is the transformation curve for a similar situation for which an infinitely large cluster was simulated by not removing atoms from the first level but by correcting instead for the binding energy at the half-crystal position at the center of an atomic row (see text for details). The thick arrow marks the absolute maximum of the transformation curve. The remaining arrows show that local maxima of the latter curve tend to be higher for $i \times(i-1)+1$ atoms, with $i$ an integer, than for $i \times i+1$ atoms. The insert shows a selected region of the plot of the barrier height vs lattice misfit for a potential with $\mu=2 v=12$ containing also the critical nucleus sizes for both types of configurations. These data confirm the appearance of only (rectangle +1 )-type islands for the simulated infinite islands.

at a kink (half-crystal) position at the middle of the last atomic row of the initial monolayer island (in the limit of a very large island, this quantity gives the chemical potential of the overlayer material on the considered substrate). ${ }^{52,53}$

A close look at the transformation curve for the simulated infinite island in Fig. 4 reveals that the maxima corresponding to (rectangle +1 ) configurations, marked by arrows in the figure, are slightly but consistently higher than those for (square +1 ) configurations when considered above the overall smooth curve described by the local maxima. In this way, only the former configurations will evolve into global maxima with varying lattice misfit. This is ultimately a consequence of the fact that nuclei of rectangular shape have higher energies than those of square shape due to the square symmetry of the model geometry. This effect is more important if the absolute value of the misfit is large or the lower island is very big. Then the size $n^{*}$ of the second layer nucleus is much smaller than the number of atoms in the lower island edge and the atomistics of the latter does not play any significant role. This is further confirmed by the insert of Fig. 4, which shows the values at the maxima of the transformation curves, $\Delta G_{\max }$ as a function of the lattice misfit, together with the sizes of the critical nuclei. It can be seen that the values of the barrier heights are very similar for the finite-sized and for the simulated infinite islands, while the critical sizes of the form (square +1$)[(i \times i+1$ atoms) $]$ change to (rectangle +1$)[i \times(i-1)+1$ atoms $]$.

In summary, we have found that the work of formation of second-layer nuclei in heteroepitaxy is smallest at the corner of the first-layer island and largest at the center, in accordance with experiments. Thus thermodynamics of second-layer nucleation is compatible with the kinetics of this process as suggested by Villain. ${ }^{34}$ The critical nucleus consists of one atom in addition of a compact shape; it plays the role of a one-dimensional nucleus giving rise to a new atomic row. The compact shape is in general a rectangle with edges of $i$ and $i-1$ atoms, while square shapes can also appear if the length of the critical nucleus is comparable to the number of atoms in the edge of the original first-layer island.

\section{ACKNOWLEDGMENT}

This work was supported by the Spanish MiCInn, Project No. FIS2008-01431. *joseemilio.prieto@uam.es

†imarkov@ipc.bas.bg

${ }^{1}$ J. Tersoff and R. M. Tromp, Phys. Rev. Lett. 70, 2782 (1993).

${ }^{2}$ J. Tersoff and F. K. LeGoues, Phys. Rev. Lett. 72, 3570 (1994).

${ }^{3}$ P. Politi, G. Grenet, A. Ponchet, and J. Villain, Phys. Rep. 324, 271 (2000).

${ }^{4}$ C. Duport, C. Priester, and J. Villain, in Directions in Condensed Matter Physics, edited by Z. Zhang and M. G. Lagally (World Scientific, Singapore, 1998), Vol. 14.

${ }^{5}$ P. Müller and R. Kern, Surf. Sci. 457, 229 (2000).

${ }^{6}$ F. Much and M. Biehl, Europhys. Lett. 63, 14 (2001).

${ }^{7}$ A. C. Schindler, D. D. Vvedensky, M. F. Gyure, G. D. Sims, R. E. Caflisch, and C. Connel, in Atomistic Aspects of Epitaxial Growth, NATO Science Series edited by M. Cotrla, N. I. Papanocolau, D. D. Vvedensky, and Luc. T. Wille (Kluwer, Amsterdam, 2002), Vol. 65, p. 337.
${ }^{8}$ B. A. Joyce and D. D. Vvedensky, in Atomistic Aspects of Epitaxial Growth, NATO Science Series edited by M. Cotrla, N. I. Papanocolau, D. D. Vvedensky, and Luc. T. Wille (Kluwer, Amsterdam, 2002), Vol. 65, p. 301.

${ }^{9}$ B. Voigtländer and A. Zinner, Appl. Phys. Lett. 63, 3055 (1993).

${ }^{10}$ Y. Ebiko, S. Muto, D. Suzuki, S. Itoh, H. Yamakoshi, K. Shiramine, T. Haga, K. Unno, and M. Ikeda, Phys. Rev. B 60, 8234 (1999).

${ }^{11}$ Y.-W. Mo, D. E. Savage, B. S. Swartzentruber, and M. G. Lagally, Phys. Rev. Lett. 65, 1020 (1990).

${ }^{12}$ E. Sutter, P. Sutter, and J. E. Bernard, Appl. Phys. Lett. 84, 2262 (2004).

${ }^{13}$ K. M. Chen, D. E. Jesson, S. J. Pannycook, T. Thundat, and R. J. Warmack, Phys. Rev. B 56, R1700 (1997).

${ }^{14}$ A. Vailionis, B. Cho, G. Glass, P. Desjardins, D. G. Cahill, and J. E. Greene, Phys. Rev. Lett. 85, 3672 (2000).

${ }^{15}$ L. V. Arapkina and V. A. Yuryev, Phys. Rev. B 82, 045315 (2010).

${ }^{16}$ P. Sutter and M. G. Lagally, Phys. Rev. Lett. 84, 4637 (2000). 
${ }^{17}$ R. J. Asaro and W. A. Tiller, Metall. Trans. 3, 1789 (1972).

${ }^{18}$ M. A. Grinfeld, Sov. Phys. Dokl. 31, 831 (1986).

${ }^{19}$ D. J. Srolovitz, Acta Metall. 37, 621 (1989).

${ }^{20}$ R. M. Tromp, F. M. Ross, and M. C. Reuter, Phys. Rev. Lett. 84, 4641 (2000).

${ }^{21}$ J. Tersoff, B. J. Spencer, A. Rastelli, and H. von Känel, Phys. Rev. Lett. 89, 196104 (2002).

${ }^{22}$ C. Priester and M. Lannoo, Phys. Rev. Lett. 75, 93 (1995).

${ }^{23}$ E. Korutcheva, A. M. Turiel, and I. Markov, Phys. Rev. B 61, 16890 (2000).

${ }^{24}$ J. E. Prieto and I. Markov, Phys. Rev. B 66, 073408 (2002).

${ }^{25}$ J. E. Prieto and I. Markov, Phys. Rev. B 72, 205412 (2005).

${ }^{26}$ K. E. Khor and S. Das Sarma, Phys. Rev. B 62, 16657 (2000).

${ }^{27}$ R. Xiang, M. T. Lung, and C.-H. Lam, Phys. Rev. E 82, 0216012010.

${ }^{28}$ J. E. Prieto and I. Markov, Phys. Rev. Lett. 98, 176101 (2007).

${ }^{29}$ L. Gavioli, K. R. Kimberlin, M. C. Tringides, J. F. Wendelken, and Z. Zhang, Phys. Rev. Lett. 82, 129 (1999).

${ }^{30}$ J. Chen, M. Hupalo, M. Ji, C. Z. Wang, K. M. Ho, and M. C. Tringides, Phys. Rev. B 77, 233302 (2008).

${ }^{31}$ Z. Zhang, Q. Niu, and C.-K. Shih, Phys. Rev. Lett. 80, 5381 (1998).

${ }^{32}$ S. Stoyanov and I. Markov, Surf. Sci. 116, 313 (1982).

${ }^{33}$ I. Markov and S. Stoyanov, Contemp. Phys. 28, 267 (1987).

${ }^{34}$ J. Villain, J. Cryst. Growth 275, e2307 (2005).

${ }^{35}$ Th. Michely and J. Krug, Islands, Mounds and Atoms: Patterns and Processes in Crystal Growth Far from Equilibrium, (Springer, Berlin, 2003).

${ }^{36}$ J. Villain, J. Phys. I (France) 1, 19 (1991).
${ }^{37}$ J. Li, Modell. Simul. Mater. Sci. Eng. 11, 173 (2003).

${ }^{38}$ I. Markov and A. Trayanov, J. Phys. C 21, 2475 (1988).

${ }^{39}$ I. Markov, Phys. Rev. B 48, 14016 (1993).

${ }^{40}$ J. Shi and X. R. Qin, Phys. Rev. B 73, 121303(R) (2006).

${ }^{41}$ P. W. Murray, I. Stensgaard, E. Laegsgaard, and F. Besenbacher, Phys. Rev. B 52, R14404 (1995).

${ }^{42}$ S. I. Molina, M. Varela, D. L. Sales, T. Ben, J. Pizarro, P. L. Galindo, D. Fuster, Y. González, L. González, and S. J. Pennycook, Appl. Phys. Lett. 91, 143112 (2007).

${ }^{43}$ B. Ranguelov, M. S. Altman, and I. Markov, Phys. Rev. B 75, 245419 (2007)

${ }^{44}$ D. Walton, in Nucleation, edited by A. C. Zettlemoyer (Marcel Dekker, New York, 1969), p. 379.

${ }^{45}$ I. Markov, in Springer Handbook of Crystal Growth, edited by G. Dhanaraj, K. Byrappa, V. Prasad, and M. Dudley (Springer, Berlin, 2010), p. 17.

${ }^{46}$ D. Kashchiev, J. Chem. Phys. 129, 164701 (2008).

${ }^{47}$ R. Kaischew and I. N. Stranski, Z. Phys. Chem. B 26, 317 (1934).

${ }^{48}$ R. Kaischew and I. N. Stranski, Z. Phys. Chem. A 170, 295 (1934).

${ }^{49}$ V. V. Voronkov, Sov. Phys. Crystallogr. 15, 13 (1970).

${ }^{50}$ I. Markov, Crystal Growth for Beginners, Fundamentals of Nucleation, Crystal Growth and Epitaxy, 2nd ed. (World Scientific, Singapore, 2003), p. 283.

${ }^{51}$ R. Becker and W. Döring, Ann. Phys. 416, 719 (1935).

${ }^{52}$ W. Kossel, Nachr. Ges. Wiss. Göttingen, Math.-Phys. Kl. 135 (1927).

${ }^{53}$ I. N. Stranski, Z. Phys. Chem. 136, 259 (1928). 